\title{
Absolute stellar proper motions with reference to galaxies of the M 81 group
}

\author{
M. Odenkirchen ${ }^{1}$, P. Brosche ${ }^{1}$, F. Börngen ${ }^{2}$, H. Meusinger ${ }^{2}$, and R. Ziener ${ }^{2}$ \\ 1 Sternwarte der Universität Bonn, Auf dem Hügel 71, D-53121 Bonn, Germany \\ 2 Thüringer Landessternwarte Tautenburg, Sternwarte 5, D-07778 Tautenburg, Germany
}

Received August 22; accepted November 4, 1996

\begin{abstract}
We report on the measurement of absolute proper motions of stars of magnitude 7 to 14.5 in a $2^{\circ} \times 2^{\circ}$ field in the region of the M 81 group of galaxies. This work is part of the Bonn program for the extragalactic link of Hipparcos. The proper motions were determined from a collection of photographic plates covering the time interval from 1894 to 1994. The absolute reference system for the proper motions is represented by the galaxies M 81, M 82 and NGC 3077, for which accurate optical reference positions were obtained by means of an image cross-correlation technique. For the majority of stars, the internal proper motion accuracy is better than $1 \mathrm{mas} / \mathrm{a}$. The precision of the realization of the absolute system is found to be around $1 \mathrm{mas} / \mathrm{a}$. In total, our measurements yield absolute proper motions for 24 Hipparcos stars and positions, proper motions and photometry for 330 additional stars including a number of visual binaries and high proper motion stars. Among the latter one G-type subdwarf is identified. The results are relevant as a contribution to the Hipparcos link, but also as a database for kinematic investigations.
\end{abstract}

Key words: galaxies: M 81 - astrometry — reference systems - catalogs

\section{Introduction}

Absolute proper motions of stars and star clusters play a key role in many aspects of galactic kinematics and dynamics. Therefore, the measurement of absolute proper motions is one of the most important tasks of modern astrometry. However, the construction of an absolute reference system (be it locally or globally) with reasonable accuracy (e.g. below 1 mas/a) is a difficult subject. While the classical approach to an absolute system was based on the

Send offprint requests to: M. Odenkirchen dynamics of the solar system, the more favoured approach today is to link the observations to distant extragalactic objects, i.e. galaxies or quasars. This is usually done by means of earth-bound photographic astrometry. The work reported here is part of the Bonn extragalactic link program, which is dedicated to an extragalactic calibration of the proper motion system of the Hipparcos catalogue. A recent summary of this program is given by Tucholke et al. (1996) and Geffert et al. (1996b). The present paper focuses on special efforts to determine absolute proper motions with respect to bright galaxies beyond the local group. The use of bright galaxies as reference objects is motivated by the fact that for some of these objects a very long history of photographic observations exists. This offers the advantage of measuring proper motions with high individual accuracy. On the other hand, the extended and structured appearance of such galaxies causes difficulties in the derivation of stable reference positions. In an earlier paper (Odenkirchen \& Brosche 1995, Paper I) it was shown that the problem of complicated source structure in principle can be overcome with the method of image crosscorrelation. We first applied this method to extragalactic sources in and around M51. Here we describe the continuation of this work. We present an application of the cross-correlation method to images of the spiral galaxy M 81 and the irregular galaxies M 82 and NGC 3077 and discuss the results.

\section{Observations and measurements}

The field of the galaxy M81 has been photographically observed since the early times of stellar photography. However, there exist not as many early plates for this field as were found in the case of M51 (Paper I), and some of the oldest M81 plates were not available to us for measurement. The oldest observations that we use in this study originate from the Greenwich part of the Carte du Ciel/Astrographic Catalogue project ( $\mathrm{CdC} / \mathrm{AC})$. We selected a sample of 5 fully or partly overlapping plates 
Table 1. The plate material

\begin{tabular}{rclcl}
\hline $\begin{array}{r}\text { Plate } \\
\text { No. }\end{array}$ & Date & Telescope & $\begin{array}{r}\text { Expo } \\
{[\mathrm{min}]}\end{array}$ & Emulsion/Filter \\
\hline 1933 & 03.4 .1894 & NA Gre & $6 \& 3$ & Mawson \\
3877 & 27.2 .1898 & NA Gre & $6 \& 3$ & Ilford \\
3914 & 21.3 .1898 & NA Gre & 40 & Ilford \\
4807 & 28.1 .1900 & NA Gre & $6 \& 3$ & Ilford \\
4906 & 19.4 .1900 & NA Gre & 40 & Rocket \\
2525 & 28.2 .1910 & BR Heid & 45 & unknown \\
2526 & 28.2 .1910 & BR Heid & 45 & unknown \\
888 & 31.3 .1957 & TA Kiev & 60 & Astro Spezial/no \\
242 & 04.4 .1959 & NA Her & 40 & unknown \\
2847 & 18.2 .1969 & ST Taut & 1 & ZU2/GG13 \\
5897 & 27.3 .1971 & NA Her & 60 & IIa-O/GG13 \\
5898 & 27.3 .1971 & NA Her & 55 & IIa-O/GG13 \\
5908 & 30.3 .1971 & NA Her & 20 & IIa-O/no \\
5909 & 14.4 .1971 & NA Her & 15 & IIa-O/no \\
4668 & 21.2 .1976 & ST Taut & 4 & ZU2/GG13 \\
4669 & 21.2 .1976 & ST Taut & 1 & ZU2/GG13 \\
1532 & 15.4 .1980 & DR OHL & 20 & 103a-O/BG3 \\
1533 & 15.4 .1980 & DR OHL & 5 & 103a-O/BG3 \\
8269 & 19.3 .1993 & ST Taut & 2 & ZU21/GG13 \\
8343 & 16.5 .1993 & ST Taut & 20 & ZU21/GG13 \\
8346 & 17.5 .1993 & ST Taut & 29 & ZU21/GG13 \\
1808 & 15.3 .1993 & DR OHL & 6 & IIa-O/BG25 \\
1810 & 15.3 .1993 & DR OHL & 6 & IIa-O/BG25 \\
1847 & 18.2 .1994 & DR OHL & 60 & IIa-O/BG25 \\
\hline 4664 & 21.2 .1976 & ST Taut & 4 & IIa-D/GG11 \\
4665 & 21.2 .1976 & ST Taut & 1 & IIa-D/GG11 \\
\hline & & & & \\
\hline
\end{tabular}

$\begin{array}{ll}\text { BR Heid }= & \begin{array}{l}\text { Bruce refractor, Heidelberg-Königstuhl } \\ \left(D=0.3 \mathrm{~m}, f=2.0 \mathrm{~m}, \text { scale } 100^{\prime \prime} / \mathrm{mm}\right)\end{array} \\ \text { NA Gre }= & \begin{array}{l}\text { Normal astrograph, Greenwich } \\ \left(D=0.3 \mathrm{~m}, f=3.4 \mathrm{~m}, \text { scale } 60.0^{\prime \prime} / \mathrm{mm}\right)\end{array} \\ \text { NA Her }= & \text { same telescope, installed at Herstmonceux } \\ \text { TA Kiev }= & \begin{array}{l}\text { Toepfer astrograph, Kiev } \\ \left(D=0.4 \mathrm{~m}, f=5.5 \mathrm{~m}, \text { scale } 37.5^{\prime \prime} / \mathrm{mm}\right)\end{array} \\ \text { ST Taut = } & \begin{array}{l}\text { Tautenburg Schmidt telescope } \\ \left(D=1.34 \mathrm{~m}, f=4.0 \mathrm{~m}, \text { scale } 51.5^{\prime \prime} / \mathrm{mm}\right)\end{array} \\ \text { DR OHL = } & \begin{array}{l}\text { Double refractor, Observatorium Hoher List } \\ \left(D=0.3 \mathrm{~m}, f=5.1 \mathrm{~m}, \text { scale } 40.4^{\prime \prime} / \mathrm{mm}\right) .\end{array}\end{array}$

(3 from AC, 2 from CdC) with epochs from 1894 to $1900^{1}$. Other early observations come from a pair of plates taken with the Heidelberg Bruce refractor in the year 1910. We also collected a number of plates from intermediary epochs. They were taken at the Observatories of Kiev, Herstmonceux, Tautenburg and Hoher List and cover the time interval from 1957 to 1980 . New plates were taken in 1993 and 1994 at Tautenburg and Hoher List. A full account of the plate material is given in Table 1. Apart from the plates of the Tautenburg Schmidt, only plates from telescopes of astrograph type are used. The plate

\footnotetext{
$1 \quad$ It is worth noting that the $\mathrm{CdC}$ plates of the Greenwich zone differ from those usually found in other $\mathrm{CdC}$ zones since they carry a single 40 min exposure and they have a stronger overlap (1 quarter) between adjacent fields. Both conditions are favourable with regard to astrometry.
}

scale ranges from $40^{\prime \prime}$ to $100^{\prime \prime}$ per $\mathrm{mm}$. The field of view varies between $\left(1.5^{\circ}\right)^{2}$ and $\left(3.2^{\circ}\right)^{2}$, but the central field in which most of the plates overlap has a size of about $\left(2^{\circ}\right)^{2}$. The limiting magnitude also differs among the plates. In general, the short exposures have a limit of $12{ }^{\mathrm{m}} 0$ while the longer exposures reach at least 14.5 . The short exposures were included because they are important for establishing a link to bright catalogue stars, in particular those of the Hipparcos catalogue. The older plates in our series (epochs before 1960) have been taken without filter, yet the emulsions that were used are known to be blue-sensitive. The latter is confirmed by the results of our photometry (see Sect. 3.2). In order to avoid as far as possible systematic errors from differences in the colours of the observations, we chose only exposures in the blue passband also for later epochs. The two V plates listed at the bottom of Table 1 served to accomplish the photometry, but were not used for astrometric purposes. All plates were measured on a PDS $2020 \mathrm{GM}^{\text {plus }}$ microdensitometer at the Astronomical Institute Münster in a similar procedure as described in Paper I. The target list (total of 383 objects) was defined by objects visible on the plates of the first epoch, that is the Greenwich AC and CdC plates, and by a number of additional stars in the surroundings of the central field, which are members of either PPM (Röser \& Bastian 1991) or HIC (Turon etal. 1992). In each scan control measurements on the positional stability of the machine were made. Also, some of the plates were measured twice in different orientation and the results compared. These tests confirmed that the measuring accuracy of the machine is at the level of $1 \mu \mathrm{m}$ or slightly below.

\section{Image processing}

\subsection{Centering of the stellar images}

From the digitized images the $x-y$-positions of the stars were determined by means of a least-squares fit of a (modified) two-dimensional Gaussian. The details of this approach have already been described in Paper I. In general, the stellar images were well fitted by a spherically symmetric distribution. Images with strong elongation appeared only near the edges and corners of plates from the Greenwich normal astrograph. Extensive tests with different plates and different image models showed that the effect becomes detrimental to astrometry for distances larger than $50 \mathrm{~mm}$ from the plate center (angular distance 0 .83). The reason is that the images are not merely elongated, but have an asymmetric profile along their major axis. A fit of an asymmetric model profile did not lead to satisfactory results with regard to the position. Therefore we decided to accept measurements from these plates only within the above mentioned limit.

Depending on the brightness of the star and on the quality of the exposure, the fitting procedure gave formal 
errors for the coordinates of the image center in the range of $0.2 \mu \mathrm{m}$ to $0.6 \mu \mathrm{m}$. This "centering error" describes the goodness of the centering process but does not account for the (large-scale) measuring accuracy of the machine, the positional stability of the emulsion etc. The total accuracy of the stellar positions as derived by comparing results from different plates of the same epoch is between $1.2 \mu \mathrm{m}$ and $1.8 \mu \mathrm{m}$ per plate.

\subsection{Photometry}

Although the emphasis of this study is on proper motions, we put some effort also on the photometric evaluation of the plates, because photometry is an important complement to proper motions and for most of the fainter stars in our list dedicated photometric measurements are lacking. Eight plates from the blue passband and two additional plates taken in the visual band were selected for the determination of stellar magnitudes. The instrumental magnitudes from the the fitting procedure were transformed to standard $B$ and $V$ by means of photoelectric and CCD measurements of up to 50 stars from de Vaucouleurs et al. (1994), Doroshenko (1994), Oja (1987), Carney \& Latham (1987) and HIC. The agreement between the results from different plates was generally better than 0.1 . A comparison with independent measurements that were not used in the calibration process also gave residuals of not more than $0 .{ }^{\mathrm{m}} 1$. Therefore we assume that the accuracy of our results is at this level. From the distribution of measured magnitudes it follows that our sample of stars is (in the central part of the field) complete to about $12 \mathrm{~m} .5$ in $V$ an $13 . \mathrm{m} 5$ in $B$, while the magnitude limits of the sample are 14.5 and 15.5 respectively.

\subsection{Derivation of absolute reference positions}

Three extragalactic sources in the field around M 81 are bright enough to be visible not only on Schmidt plates but also on early astrograph exposures. They are located in the center of M 81 and in the inner parts of NGC 3077 and M 82 (central $30^{\prime \prime}$ to $120^{\prime \prime}$ ). The center of M 81 is an exceptionally bright source with a rather high degree of symmetry, i.e. the isophotes can be represented by a system of concentric ellipses. In order to see what can be achieved with a normal centering method, we applied a fit of an elliptical model distribution to the images of this source. Depending on the quality of the individual images, internal accuracies between 0.3 and $0.7 \mu \mathrm{m}$ for the position of the object center were obtained. This indicates that the fitting method is indeed applicable in this case.

The other two sources show complex and multiple structures. In M 82 we find two groups of knots (commonly interpreted as starburst regions), which are separated by a strong dust filament. Here, a fit of a suitable model func- tion is practically impossible. However, the positional information contained in the images of these sources is accessible through direct comparison between the images by means of maximum cross-correlation. The practical implementation of this method works as follows: 1) All images are rebinned to a common plate scale. 2) The essential structure (the "signal") is extracted on all plates in images of equal size. 3) One image of the series is chosen as a "reference image" and an arbitrary point in it (e.g. the center of the frame) is selected as "reference point". 4) Each image is shifted into the coordinate domain of the reference image. The cross-correlation between the images is calculated as a function of the shift vector. The optimum shift, at which the crosscorrelation is at maximum, is searched $^{2}$.5) Finally, with the optimum shift vectors the location of the reference point is transferred from the reference plate to all other plates. The cross-correlation (see Eq. (2) of Paper I) can be calculated in different ways. One can either consider the images as step functions and evaluate $C$ only for shifts, which are integer multiples of one pixel in $x$ and $y$. Then, the maximum of $C$ must be found by interpolating in the grid of values of $C$, for instance with a 2nd order polynomial. Or one can consider the images as continous functions, for instance with bilinear interpolation between adjacent pixel values. This allows to evaluate $C$ with continously varying shifts and to find the maximum of $C$ directly.

Both possibilities were tested on the images of $\mathrm{M} 81$, M 82 and NGC 3077. The positions of the maximum of $C$ from both methods were found to be consistent on the level of 0.04 pixel or $0.4 \mu \mathrm{m}$ in each coordinate (rms deviation). We also tested how much the results of the crosscorrelation depend on the particular choice of the reference image and the frame size. Again, the deviations between the different results were found to be small and of minor importance in the total positional error budget. The final results of both the cross-correlation method and the fitting method (in the case of M 81) are summarized in Table 2. The figures given there were obtained at the end of the plate reduction described in the next section. An estimate of the positional accuracy can be read off from the dispersions $\sigma, \sigma^{\prime}$ around the mean trend. For M 81 and M 82e the positions from the different plates agree on the level of 0 .' 15 , while for NGC 3077 the dispersion in the positions is somewhat larger and reaches 0 ' $^{\prime} 20$ to $0{ }^{\prime \prime} 24$. The latter is probably not due to the source structure, but can be understood as a consequence of the weakness of the source.

\footnotetext{
2 Rotations between the images can a priori be excluded by proper orientation of the plate in the scan.
} 


\section{Data reduction and results}

\subsection{Iterative overlap solution}

In order to derive proper motions, the $x$ - $y$-positions measured on different plates were "reduced" to the system of an external stellar reference catalogue by means of a simultaneous adjustment of all plates. The solution was approached with an iterative reduction scheme, in which single plate adjustments and astrometric parameter determinations were alternated until convergence in the plate constants and in the positions and proper motions of all objects was reached (iterative overlap algorithm). Two solutions were tried, one with the PPM catalogue and one with data from the preliminary catalogue $\mathrm{H} 37^{3}$. Here we describe the second solution, which we regard the more interesting and preferable one. 34 stars from H37 were available in the field and served as reference stars. In the initial step of the reduction only the plates with the largest field size and hence the largest number of reference stars were used (Schmidt and Bruce plates, $N \geq 22$ reference stars). The plate-to-catalogue transformations were made with either gnomonic (astrograph) or equidistant (Schmidt) projection and with a full 2nd order polynomial plate model. Terms of 3rd order proved to be dispensable. With five iteration steps a stable solution was reached. The $x-y$-residuals of this solution are between $1.0 \mu \mathrm{m}$ and $2.0 \mu \mathrm{m}$ (rms per plate), depending on plate quality. The internal errors of the proper motions are generally at or below the level of 1 mas/a, except for very faint stars or stars with less than 6 observations.

\subsection{Fictitious motions of the galaxies}

The measurements of the extragalactic sources were not included in the plate adjustment procedure, but were introduced only in the final step of the reduction. Since the stellar reference frame to which the solution refers is not a priori an absolute frame, proper motions were assigned to the galaxies in the same way as to the stars. However these proper motions are fictitious, because (apart from measuring errors) they indicate the deviation of the stellar frame from the extragalactic frame. Thus they provide the key for transferring the stellar proper motions to the absolute frame. In Table 2 we give the individual proper motion values derived for the three different sources. It is not surprising that the highest internal proper motion accuracy is achieved with the center of M 81. The results from the fitting approach are in good agreement with those from cross-correlation and both methods yield essentially the same internal accuracy of 1 mas/a. In the case of M 82e the internal accuracy of the fictitious proper motions is on the level of $1.5 \mathrm{mas} / \mathrm{a}$. This is mainly due to a lower

3 H37 contains data from the Hipparcos 37-month-solution. It was provided to us by permission of the Hipparcos Science Team.
Table 2. Fictitious proper motions of extragalactic sources obtained with different methods

\begin{tabular}{llccccccc}
\hline $\begin{array}{c}\text { Source \& } \\
\text { method }\end{array}$ & \multicolumn{2}{c}{$\mu$} & $\epsilon_{\mu}$ & \multicolumn{2}{c}{$\mu^{\prime}$} & $\epsilon_{\mu^{\prime}}$ & $N$ & \multicolumn{2}{c}{$\sigma \sigma^{\prime}$} \\
\hline M 81n & $g f$ & -0.8 & 1.0 & -0.3 & 1.1 & 19 & 0.14 & 0.15 \\
& $c c$ & -1.1 & 1.1 & -0.9 & 0.7 & 19 & 0.16 & 0.10 \\
M 82e & $c c$ & -0.1 & 1.4 & -2.6 & 1.3 & 15 & 0.15 & 0.13 \\
N 3077 & $c c$ & +2.1 & 2.1 & -0.3 & 1.7 & 16 & 0.24 & 0.20 \\
\hline
\end{tabular}

$g f=$ with positions from fit of a modified Gaussian

$c c=$ with positions from cross-correlation

$\mu, \epsilon_{\mu}=$ proper motion $\mu_{\alpha} \cos \delta$ in $\alpha$ and its rms error

$\mu^{\prime}, \epsilon_{\mu^{\prime}}=$ proper motion in $\delta$ and its rms error

$N=$ Number of photographic observations

$\sigma, \sigma^{\prime}=$ dispersion of positions around mean trend.

number of observations. In the right ascension component the proper motion agrees well with that of M 81n within the formal errors, while in the declination component the deviation between both results exceeds the formal error by a factor of 1.5. A similar statement holds for the proper motions of NGC 3077. Here, the declination component is consistent with the results on M 81n, but the right ascension component deviates at the margin of the combined error bar. The conclusion is that the formal errors somewhat overestimate the true random accuracy or that a position-dependent systematic error remains, which can however not be analysed here.

From the individual fictitious motions the local shift between absolute frame and stellar frame (zero point correction) was determined by averaging the two results for M 81n and taking a weighted mean over the three sources with weights according to the formal errors. The result is $\left(\mu, \mu^{\prime}\right)=(-0.2,-1.2) \mathrm{mas} / \mathrm{a}$. This correction was subtracted from the results for the stars in order to obtain absolute proper motions. From the deviations between the individual ficticious proper motions and the above given mean we estimate that the absolute system is determined with an accuracy of about 1 mas/a. This is confirmed by comparison with results from other link fields of the Bonn programme (see Sect. 4.4).

\subsection{Absolute proper motions of the stars}

For $95 \%$ of the stars the reduction procedure gave proper motions with formal errors below $1.5 \mathrm{mas} / \mathrm{a}$. The formal errors are distributed around a median of $0.6 \mathrm{mas} / \mathrm{a}$ in each coordinate. The external accuracy may be somewhat lower, but at the moment this can hardly be examined because there are (except from Hipparcos) no other measurements on our sample of stars with a similar level of accuracy. Nevertheless, it is worthwhile to compare our results to other proper motion catalogues. This is done 
Table 3. Comparison of our (absolute) proper motions with proper motions from other catalogues

\begin{tabular}{lcccccc}
\hline $\begin{array}{l}\text { this work } \\
\text { minus }\end{array}$ & $\begin{array}{c}\text { No. of } \\
\text { stars }\end{array}$ & $\begin{array}{c}\overline{\Delta \mu} \\
{[\mathrm{mas} / \mathrm{a}]}\end{array}$ & $\begin{array}{c}\sigma_{\Delta \mu} \\
{[\mathrm{mas} / \mathrm{a}]}\end{array}$ & $\begin{array}{c}\sigma_{\Delta \mu^{\prime}} \\
\text { Ref. }\end{array}$ \\
\hline NPM & 12 & +0.2 & 4.5 & +0.0 & 5.7 & $(1)$ \\
PPM & 49 & -0.8 & 5.6 & -1.7 & 4.5 & $(2)$ \\
PPM, HPS & 11 & +0.1 & 2.9 & -1.2 & 2.9 & $(2)$ \\
ACRS & 31 & +5.2 & 8.7 & -2.7 & 6.4 & $(3)$ \\
\hline
\end{tabular}

$\mu, \mu^{\prime}=$ proper motions $\mu_{\alpha} \cos \delta$ and $\mu_{\delta}$ in $\alpha$ and $\delta$

$\bar{\Delta}=$ mean of individual differences

$\sigma_{\Delta}=$ rms deviation from the mean

(1) Klemola et al. (1993a)

(2) Röser \& Bastian (1991)

(3) Corbin \& Urban (1991).

in Table 3. For a test of the absolute proper motion zero point the best catalogue to compare with is that of the Lick Northern Proper Motion program (NPM, Klemola et al. 1993a,b). A comparison of 12 stars in common with the NPM yields very satisfactory agreement between the two extragalactically calibrated systems. Also, the dispersions in the proper motion differences to NPM fit well to the expected random error of NPM proper motions of 5 mas/a. Dispersions of similar size are found when comparing to 49 stars from the PPM catalogue. They slightly exceed the nominal error of $4.3 \mathrm{mas} /$ a quoted in the PPM. The mean differences relative to PPM are marginally significant, but stay below 2 mas/a. Both the mean and the dispersion of the proper motion differences decrease when the comparison is confined to stars of the PPM High Precision Subset (HPS). We conclude that in the field under consideration the PPM deviates from an absolute system by less than $1.5 \mathrm{mas} / \mathrm{a}$. Totally different results are obtained in comparison to 31 stars from the ACRS (Corbin \& Urban 1991). There we find mean proper motion differences of $+5.2 \mathrm{mas} / \mathrm{a}$ and $-2.7 \mathrm{mas} / \mathrm{a}$ and dispersions which are clearly above the nominal ACRS random error of $4.7 \mathrm{mas} / \mathrm{a}$. Hence a local disturbance in the proper motions of the ACRS must be assumed ${ }^{4}$.

\subsection{Hipparcos stars}

Special attention needs to be given to the Hipparcos stars. Table 4 presents the proper motion results for a subsample of 24 Hipparcos stars, for which at least 8 photographic observations from different epochs were available in the reduction. In most cases the proper motions are based on

\footnotetext{
4 Note that a global comparison between ACRS and PPM by Soma (1993) shows a maximum of systematic deviation in the declination ring of $+65^{\circ}$ to $+70^{\circ}$, in which our field is located. Size and sign of this zonal error agree with the deviation described above.
}

Table 4. Photographically determined absolute proper motions of Hipparcos stars

\begin{tabular}{lrrrrr}
\hline HIC & \multicolumn{2}{c}{$\mu$} & $\epsilon_{\mu}$ & \multicolumn{2}{c}{$\mu^{\prime}$} \\
No. & \multicolumn{2}{c}{$\epsilon_{\mu^{\prime}}$} & $N$ \\
\hline 47443 & -10.1 & 0.9 & -3.1 & 0.7 & 8 \\
47594 & -67.1 & 0.8 & -68.4 & 0.6 & 11 \\
47620 & -671.0 & 0.7 & -269.3 & 0.5 & 10 \\
47650 & -671.1 & 1.0 & -264.9 & 0.7 & 8 \\
47737 & -12.2 & 1.1 & +10.8 & 0.8 & 8 \\
47801 & +17.3 & 0.3 & +14.4 & 0.5 & 11 \\
47838 & -14.7 & 0.7 & +9.8 & 1.1 & 8 \\
48139 & -26.4 & 0.4 & -327.9 & 0.3 & 13 \\
48285 & -29.3 & 0.3 & -11.4 & 0.6 & 13 \\
48486 & -6.8 & 0.5 & -12.1 & 0.5 & 19 \\
48573 & -124.0 & 0.4 & -103.2 & 0.5 & 21 \\
$48635^{*}$ & -63.5 & 0.8 & -61.7 & 0.5 & 18 \\
$48635^{*}$ & -62.6 & 1.1 & -62.3 & 0.5 & 17 \\
$48639^{*}$ & -7.9 & 0.5 & -3.7 & 0.5 & 22 \\
48691 & -9.1 & 0.5 & +17.6 & 0.8 & 18 \\
48803 & -8.0 & 0.9 & +0.2 & 1.7 & 11 \\
48813 & -44.6 & 0.4 & -38.2 & 0.6 & 19 \\
49121 & -43.8 & 0.7 & -24.6 & 0.5 & 19 \\
49193 & -30.5 & 0.5 & -8.1 & 0.5 & 24 \\
$49230^{*}$ & -29.8 & 0.7 & -23.3 & 0.7 & 20 \\
49392 & +10.8 & 0.5 & +3.0 & 0.4 & 11 \\
49497 & +2.4 & 0.9 & -8.2 & 0.6 & 16 \\
$49596^{*}$ & -36.4 & 0.7 & -18.8 & 0.7 & 10 \\
49649 & +11.1 & 1.0 & +21.3 & 0.5 & 10 \\
\hline
\end{tabular}

HIC = Hipparcos Input Catalogue (Turon et al. 1992)

$* \quad=$ (member of) known binary system, not in H37.

11 to 20 photographic measurements. The internal proper motion accuracy ranges between 0.3 and 1.1 mas/a (in one case $1.7 \mathrm{mas} / \mathrm{a}$ ). Thus the results reach at least the same level of internal accuracy as the Hipparcos H37 data and on the average go beyond this level. Although the results of the reduction are not completely independent from the Hipparcos data (because H37 was used as reference catalogue) it is interesting to examine the degree of accordance between the proper motions from photography and those from H37. We find that (apart from the offset between absolute and stellar frame) the proper motion values generally differ by less than 3 mas/a and that the rms of the differences is about $1.6 \mathrm{mas} / \mathrm{a}$. However, one single case ( HIC 48691) of striking disparity is noticed, in which the proper motion differences exceed the above mentioned rms by factors of 2.8 and 4 . This case is displayed in Fig. 1. The discordance may be explained by the fact, that the photographic measurements record the long-term motion whereas Hipparcos gives the instantaneous motion (during a mission of 3.5 years). Both can be different if the object is an unresolved binary and its photocenter is affected by orbital motion. The occurence of this kind of cosmic error in the proper motions of Hipparcos has recently been discussed by Wielen (1995) and by Brosche et al. (1995). According to Bernstein (1996, private 
communication) HIC 48691 yields a significantly increased $\chi^{2}$ in the Hipparcos data reduction, which suggests that it is indeed not a single star. In order to confirm the hypothesis of orbital motion, it would be desirable to have further evidence of the binarity, for instance from speckle interferometry.

Five stars from Table 4 (marked by an asterisk) are known as members of binary systems and were therefore not contained in the provisional catalogue H37. Hence they were not used here as reference stars. At a later stage they may provide an additional check between the photographic measurements and Hipparcos. However, care has to be taken when comparing the results because three of these systems are not photographically resolved.

Owing to the strong global rigidity of the Hipparcos system, our absolute proper motions can be linked to absolute proper motions obtained for Hipparcos stars in other fields of the sky and can be combined with them into one mean absolute system. This was done with stars from 12 different link fields of the Bonn programme (see Tucholke et al. 1996). The residuals from the resulting mean system give evidence of the reliability of our absolute proper motion zero point. The local absolute system determined from the galaxies M 81, M 82 and NGC 3077 agrees with the mean system of all link fields to within -0.9 and +0.2 mas/a, confirming the estimate given in Sect. 4.2.

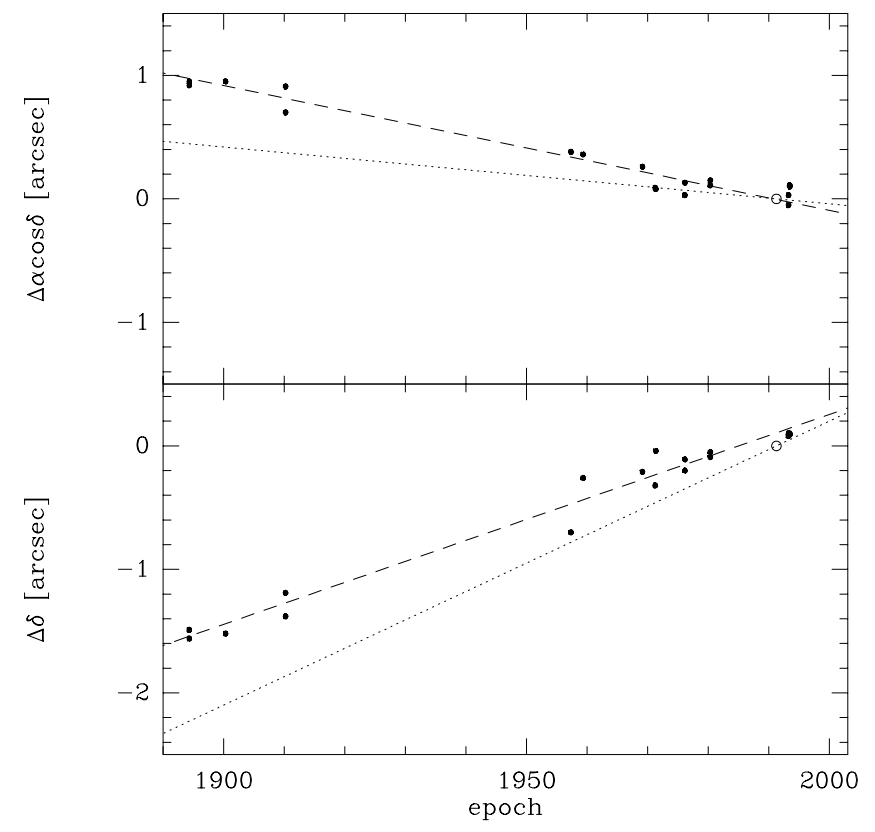

Fig. 1. Disparity in the results on HIC 48691. The points give photographically measured positions, the open circle marks the mean position from Hipparcos. The dashed line shows the mean motion according to the points, whereas the dotted line shows an extrapolation with the proper motion measured by Hipparcos

\subsection{Double stars and common proper motion pairs}

We identified in the sample of target stars six visual binaries and two common proper motion pairs. Our data for these systems are shown in Table 5 . The visual pairs were selected according to the proximity of their components ${ }^{5}$. The proper motion pairs were identified by the fact that the components show parallel motion $\left(\Delta \mu_{\text {tot }} / \mu_{\text {tot }}<10 \%\right)^{6}$ at an angular distance of ordinary neighbours in the field. While two of the pairs are well-known, the others (with anonymous component $B$ in Table 5) have so far not been mentioned in double star catalogues. The physical nature of the pairs was tested by means of proper motions and photometry. Assuming main sequence stars, we used the photometry to derive estimates for the distance of the components and also for their mass. The proper motion difference was transformed into a relative tangential space velocity and this was compared to an estimate of the orbital velocity (cf. Brosche et al. 1992). The result of this test is that Nos. 2, 3 and 5 are very likely physical binaries whereas Nos. 4, 6 and 7 should be optical pairs. However, with No. 7 there is some doubt wether this is not a physical pair as well. In the case of No. 1 the physical nature is known directly from trigonometric parallax measurements. No. 8 is a very interesting case, because the components seem to be at the same distance of about $60 \mathrm{pc}$, but as a pair they would have an extreme separation of $40500 \mathrm{AU}$ (cf. Odenkirchen $\&$ Brosche 1996). For a more decisive statement about the nature of this pair radial velocities are needed.

\subsection{High proper motion stars}

For the majority of stars in our sample $(\approx 85 \%)$ the total proper motion is below 50 mas/a. About $60 \%$ of the stars have $\mu_{\text {tot }} \leq 20$ mas/a. In this sense we regard objects with $\mu_{\text {tot }} \geq 100$ mas/a as high proper motion stars (HPM). Nine such exceptional cases were found and are listed in Table 6. Five of them occur in the HPM surveys of Luyten $(1961,1976,1979)$ and Giclas et al. (1971), since their total proper motion is larger than 200 mas/a. The pair Gl 360/362 has a total proper motion as large as $725 \mathrm{mas} / \mathrm{a}$. This is mostly due to the small distance of only $12 \mathrm{pc}$ (trig. parallax $=00^{\prime \prime} 085$ ), from which a tangential space velocity of $40 \mathrm{~km} \mathrm{~s}^{-1}$ follows. By means of our two color photometry some conclusions about distance and tangential velocity can be drawn also for the other HPM stars of Table 6. Taking into account as far as available also spectral type or trigonometric parallax measurements we derived for Nos. 4 and 5 tangential space velocities around $45 \mathrm{~km} \mathrm{~s}^{-1}$ and for Nos. 3 and 8

5 The limit was set at $20^{\prime \prime}$ since for separations below this value a clear excess over the expected number of random pairs was observed.

${ }^{6} \mu_{\mathrm{tot}}=\sqrt{\mu^{2}+\mu^{\prime 2}}$. 
Table 5. Photographic measurements of double stars and common proper motion pairs

\begin{tabular}{|c|c|c|c|c|c|c|c|c|c|c|c|c|c|}
\hline No. & Name & $\begin{array}{c}\alpha_{2000} \\
{[\mathrm{~h} \mathrm{~m} \mathrm{~s}]}\end{array}$ & 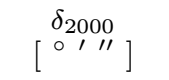 & {$\left[\begin{array}{r}\mu \\
\text { mas }\end{array}\right.$} & $\epsilon_{\mu}$ & $\begin{array}{c}\mu^{\prime} \\
{[\text { mas }}\end{array}$ & $\epsilon_{\mu^{\prime}}$ & {$\left[\begin{array}{r}\rho \\
{["]}\end{array}\right.$} & {$\left[\begin{array}{r}\theta \\
\left.{ }^{\circ}\right]\end{array}\right.$} & ${ }^{B} \mathrm{ma}$ & 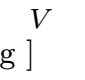 & $t$ & $n$ \\
\hline $\begin{array}{r}1 \mathrm{~A} \\
\mathrm{~B}\end{array}$ & $\begin{array}{ll}\text { Gl } & 360 \\
\text { Gl } & 362\end{array}$ & $\begin{array}{lll}09 & 42 & 34.84 \\
09 & 42 & 51.73\end{array}$ & $\begin{array}{lll}+70 & 02 & 02.0 \\
+70 & 02 & 21.9\end{array}$ & $\begin{array}{l}-671.0 \\
-671.1\end{array}$ & $\begin{array}{l}0.7 \\
1.0\end{array}$ & $\begin{array}{l}-269.3 \\
-264.9\end{array}$ & $\begin{array}{l}0.5 \\
0.7\end{array}$ & 88.7 & 77.2 & $\begin{array}{l}12.02 \\
12.73\end{array}$ & $\begin{array}{l}10.48 \\
11.20\end{array}$ & 1965 & 8 \\
\hline $\begin{array}{r}2 \mathrm{~A} \\
\mathrm{~B}\end{array}$ & $\begin{array}{l}\text { anon. } \\
\text { anon. }\end{array}$ & $\begin{array}{lll}09 & 54 & 39.81 \\
09 & 54 & 40.70\end{array}$ & $\begin{array}{l}+683637.5 \\
+683635.2\end{array}$ & $\begin{array}{l}+20.8 \\
+19.7\end{array}$ & $\begin{array}{l}1.0 \\
1.4\end{array}$ & $\begin{array}{l}-16.6 \\
-18.1\end{array}$ & $\begin{array}{l}0.6 \\
0.9\end{array}$ & 5.40 & 114.5 & $\begin{array}{l}13.58 \\
13.62\end{array}$ & $\begin{array}{l}12.63 \\
12.68\end{array}$ & 1960 & 12 \\
\hline $\begin{array}{r}3 \mathrm{~A} \\
\mathrm{~B}\end{array}$ & $\begin{array}{l}\text { ADS } 7565 \\
\text { ADS } 7565\end{array}$ & $\begin{array}{lll}09 & 55 & 02.69 \\
09 & 55 & 01.01\end{array}$ & $\begin{array}{l}+685622.4 \\
+685622.4\end{array}$ & $\begin{array}{l}-62.6 \\
-63.5\end{array}$ & $\begin{array}{l}1.1 \\
0.8\end{array}$ & $\begin{array}{l}-62.3 \\
-61.7\end{array}$ & $\begin{array}{l}0.5 \\
0.4\end{array}$ & 8.92 & 272.5 & $\begin{array}{l}11.35 \\
11.40\end{array}$ & $\begin{array}{l}10.49 \\
10.57\end{array}$ & 1960 & 12 \\
\hline $\begin{array}{r}4 \mathrm{~A} \\
\mathrm{~B}\end{array}$ & $\begin{array}{l}\text { anon. } \\
\text { anon. }\end{array}$ & $\begin{array}{lll}09 & 57 & 46.54 \\
09 & 57 & 48.09\end{array}$ & $\begin{array}{l}+682929.0 \\
+682942.2\end{array}$ & $\begin{array}{r}-26.4 \\
-4.3\end{array}$ & $\begin{array}{l}0.6 \\
0.6\end{array}$ & $\begin{array}{r}-18.5 \\
-2.4\end{array}$ & $\begin{array}{l}0.6 \\
0.8\end{array}$ & 14.81 & 31.4 & $\begin{array}{l}13.18 \\
14.71\end{array}$ & $\begin{array}{l}12.55 \\
14.08\end{array}$ & 1963 & 14 \\
\hline $\begin{array}{r}5 \mathrm{~A} \\
\mathrm{~B}\end{array}$ & $\begin{array}{l}\text { anon. } \\
\text { anon. }\end{array}$ & $\begin{array}{lll}09 & 59 & 05.06 \\
09 & 59 & 05.09\end{array}$ & $\begin{array}{l}+684726.2 \\
+684745.1\end{array}$ & $\begin{array}{l}-1.7 \\
-5.2\end{array}$ & $\begin{array}{l}0.8 \\
0.7\end{array}$ & $\begin{array}{l}+38.9 \\
+41.9\end{array}$ & $\begin{array}{l}0.4 \\
0.9\end{array}$ & 18.80 & 0.8 & $\begin{array}{l}10.65 \\
13.43\end{array}$ & $\begin{array}{r}9.94 \\
12.05\end{array}$ & 1966 & 17 \\
\hline $\begin{array}{r}6 \mathrm{~A} \\
\mathrm{~B}\end{array}$ & $\begin{array}{l}\text { anon. } \\
\text { anon. }\end{array}$ & $\begin{array}{lll}10 & 00 & 28.73 \\
10 & 00 & 25.54\end{array}$ & $\begin{array}{l}+694251.5 \\
+694249.1\end{array}$ & $\begin{array}{r}-0.3 \\
+12.5\end{array}$ & $\begin{array}{l}0.5 \\
0.6\end{array}$ & $\begin{array}{l}-1.0 \\
+6.8\end{array}$ & $\begin{array}{l}0.5 \\
0.4\end{array}$ & 17.30 & 260.9 & $\begin{array}{l}13.08 \\
13.74\end{array}$ & $\begin{array}{l}11.96 \\
13.40\end{array}$ & 1962 & 19 \\
\hline $\begin{array}{r}7 \mathrm{~A} \\
\mathrm{~B}\end{array}$ & $\begin{array}{l}\text { anon. } \\
\text { anon. }\end{array}$ & $\begin{array}{lll}10 & 01 & 32.48 \\
10 & 01 & 33.50\end{array}$ & $\begin{array}{l}+683635.3 \\
+683634.8\end{array}$ & $\begin{array}{l}-28.7 \\
-24.6\end{array}$ & $\begin{array}{l}1.3 \\
1.3\end{array}$ & $\begin{array}{l}-3.6 \\
-5.7\end{array}$ & $\begin{array}{l}0.8 \\
0.6\end{array}$ & 5.43 & 94.7 & $\begin{array}{l}13.95 \\
15.84\end{array}$ & $\begin{array}{l}13.47 \\
14.86\end{array}$ & 1967 & 8 \\
\hline $\begin{array}{r}8 \mathrm{~A} \\
\mathrm{~B}\end{array}$ & $\begin{array}{l}\text { ADS } 7611 \\
\text { anon. }\end{array}$ & $\begin{array}{lll}10 & 02 & 56.39 \\
10 & 01 & 06.91\end{array}$ & $\begin{array}{l}+684708.8 \\
+685232.2\end{array}$ & $\begin{array}{l}-29.8 \\
-32.2\end{array}$ & $\begin{array}{l}0.7 \\
0.4\end{array}$ & $\begin{array}{l}-23.3 \\
-21.6\end{array}$ & $\begin{array}{l}0.7 \\
0.5\end{array}$ & 676.4 & 298.6 & $\begin{array}{r}8.31 \\
11.73\end{array}$ & $\begin{array}{r}7.76 \\
10.65\end{array}$ & 1960 & 20 \\
\hline
\end{tabular}

$\alpha, \delta_{2000}=$ Right ascension and declination for equinox and epoch $\mathrm{J} 2000.0$

$\rho, \theta \quad=$ Angular separation and position angle between components

$t, n=$ mean epoch and number of observations of $\rho$ and $\theta$

$B, V=$ brightness in $B$ and $V$ as derived from photographic measurements

Other symbols same as in previous tables.

Table 6. Photographic measurements of high proper motion stars $\left(\mu_{\mathrm{tot}} \geq 100\right.$ mas/a)

\begin{tabular}{|c|c|c|c|c|c|c|c|c|c|c|c|}
\hline No. & Name & $\begin{array}{c}\alpha_{2000} \\
{[\mathrm{~h} \mathrm{~m} \mathrm{~s}]}\end{array}$ & $\begin{array}{c}\delta_{2000} \\
{\left[\begin{array}{lll}0 & 1 & 11\end{array}\right]}\end{array}$ & $\begin{array}{c}\mu \\
{[\text { mas }}\end{array}$ & $\epsilon_{\mu}$ & $\begin{array}{c}\mu^{\prime} \\
{[\text { mas }}\end{array}$ & $\epsilon_{\mu^{\prime}}$ & ${ }^{B}$ & $V$ & $N$ & other designations \\
\hline 1 & Gl 360 & 094234.84 & +700202.0 & -671.0 & 0.7 & -269.3 & 0.5 & 12.02 & 10.48 & 10 & LHS 2176, G 235-035 \\
\hline 2 & Gl 362 & 094251.73 & +700221.9 & -671.1 & 1.0 & -264.9 & 0.7 & 12.73 & 11.20 & 8 & LHS 2178, G 235-036 \\
\hline 3 & $\mathrm{BD}+70578$ & 094849.83 & +694555.6 & -26.4 & 0.4 & -327.9 & 0.3 & 10.78 & 9.56 & 13 & LTT 12598, G 235-041 \\
\hline 4 & $\mathrm{BD}+70586$ & 095426.77 & +692700.4 & -124.0 & 0.4 & -103.2 & 0.5 & 10.14 & 9.35 & 21 & \\
\hline 5 & anon. & 095514.92 & +693614.4 & -102.3 & 0.6 & -45.2 & 0.5 & 11.51 & 10.65 & 21 & \\
\hline 6 & $\mathrm{AC}+704348$ & 095640.95 & +693629.0 & -171.0 & 0.6 & -142.9 & 0.5 & 12.97 & 12.36 & 19 & \\
\hline 7 & anon. & 100156.94 & +681750.5 & -103.0 & 0.6 & -25.8 & 0.5 & 12.00 & 11.35 & 15 & \\
\hline 8 & $\mathrm{BD}+69558$ & 100832.63 & +682614.9 & -263.3 & 0.7 & -138.3 & 0.9 & 9.61 & 8.77 & 7 & LTT 12708, G 235-057 \\
\hline 9 & anon. & 100851.79 & +69 1635.5 & +195.9 & 0.9 & +16.9 & 0.6 & 12.88 & 11.38 & 11 & \\
\hline
\end{tabular}

$\mu_{\mathrm{tot}}=\sqrt{\mu^{2}+\mu^{\prime 2}} ;$ other symbols same as in previous tables.

velocity values of about $65 \mathrm{~km} \mathrm{~s}^{-1}$. For No. 7 we obtained in the same way an estimated distance of $190 \mathrm{pc}$ and hence a tangential space velocity of $95 \mathrm{kms}^{-1}$. If our assumptions hold, this object must be attached to the class of high velocity stars. For No. 9 our photometry yields $B-V=$ 1. 50 and thereby a distance of only 20 pc. However, with a photometric error of $0{ }^{\mathrm{m}} 1$ in the color index the distance estimate can rise to about $35 \mathrm{pc}$. The tangential space velocity would be $19 \mathrm{~km} \mathrm{~s}^{-1}$ or $33 \mathrm{~km} \mathrm{~s}^{-1}$ respectively ${ }^{7}$. In the case of No. 6 the proper motions reveal that the assump-

\footnotetext{
$\overline{7}$ Note that the direction of the proper motion vector is rather peculiar as compared to the other stars (positive $\mu$ and $\mu^{\prime}$ ).
}

tion of an ordinary main sequence star cannot be true, because it would lead to an unrealistically high space motion. On the other hand, the assumption that it is a white dwarf is also not very convincing, because it would place the object within 5 pc from the sun. Instead we conclude, that this star is most likely a G-type subdwarf. An estimated absolute luminosity of $M_{V}=6.7$ yields a distance of about $140 \mathrm{pc}$ and a tangential space velocity around $145 \mathrm{~km} \mathrm{~s}^{-1}$ relative to the sun. This is a typical result for a star belonging to the halo population. 


\section{Summary and conclusions}

Once more, the results of this paper reveal that at the dawn of the age of satellite astrometry early photographic observations are still a valuable source for measuring proper motions of fainter stars. With a modern measuring machine and with the new reference system established by Hipparcos the full wealth of positional information preserved in these plates can today be unveiled (see also Geffert et al. 1996a). The internal accuracy of the proper motions derived in this study is at the same level as or even better than the accuracy achieved with Hipparcos itself.

It was also shown that astrometric results of good accuracy can be obtained for non-pointlike extragalactic objects, if the intrinsic structure of the source is evaluated by means of a cross-correlation method. The absolute reference frame that was set up in this way by three sources in M 81, M 82 and NGC 3077 proved to be absolute at the level of $1 \mathrm{mas} / \mathrm{a}$. This was confirmed in comparison with the Lick NPM catalogue and with the mean absolute system of the Bonn Extragalactic Link program. We expect that in future the cross-correlation technique will be relevant especially for the astrometric reduction of CCD observations as obtained for example at modern transit telescopes.

Together with results from other link fields our absolute proper motions can make an important contribution to the extragalactic link of the Hipparcos system. However we pointed out that the Hipparcos results may not be fully compatible with our photographic measurements because of the influence of orbital motion.

The discussion of double stars and high proper motion stars has shown that the combination of proper motions and photometry bears a potential for investigating kinematic questions. This will be the subject of a later paper.

The complete catalogue of absolute proper motions and photometry for the field around M 81 as well as a revised version of the catalogue of proper motions in the neighbourhood of M 51 is available on request.

Acknowledgements. We thank R. Catchpole from the Royal Greenwich Observatory Cambridge, V.S. Kislyuk from the Main Astronomical Observatory Kiev and G. Klare from the Landessternwarte Heidelberg, who kindly provided plate material for this project. We also thank the Astronomisches Institut Münster for generous allocation of PDS scan time and H.-J. Tucholke for scanning some of the plates. We thank the Hipparcos Science Team for the contribution of H37 data. Finally, we gratefully acknowledge financial support from the Bundesministerium für Forschung und Technologie (FKZ 50O09101). This research has made use of the SIMBAD database, operated at CDS, Strasbourg, France.

\section{References}

Brosche P., Denis-Karafistan A.I., Sinachopoulos D., 1992, A\&A 253, 113

Brosche P., Tucholke H.-J., Odenkirchen M., 1995, AN 316, 35

Carney B.W., Latham D.W., 1987, AJ 93, 116

Corbin T.E., Urban S.E., 1991, Astrographic Catalogue Reference Stars, NSSDC

de Vaucouleurs G., Corwin H.G., Skiff B.A., 1994, PASP 106, 156

Doroshenko V.T., 1994, Astron. Lett. 20, 792

Geffert M., Bonnefond P., Maintz G., Guibert J., 1996a, A\&AS 118,277

Geffert M., Klemola A.R., Hiesgen M., Schmoll J., 1996b, A\&AS (submitted)

Giclas H.L., Burnham R., Thomas N.G., 1971, Lowell Proper Motion Survey, Lowell Observatory, Flagstaff

Gliese W., 1969, Veröff. Astron. Recheninstitut No. 22, Heidelberg

Hanson R.B., 1987, AJ 94, 409

Klemola A.R., Hanson R.B., Jones B.F., 1993a, NPM1 Catalog, NSSDC

Klemola A.R., Hanson R.B., Jones B.F., 1993b, NPM1 Reference Galaxies, NSSDC

Luyten W.J., 1961, A Catalog of 7127 Stars in the Northern Hemisphere with Proper Motions exceeding 0.' 2 annually, Lund, Minneapolis

Luyten W.J., 1976, LHS Catalogue, Univ. Minnesota, Minneapolis

Luyten W.J., 1979, NLTT Catalogue, Univ. Minnesota, Minneapolis

Röser S., Bastian U., 1991, PPM Star Catalogue. Spektrum Akademischer Verlag, Heidelberg

Odenkirchen M., Brosche P., 1995, A\&A 302, 915

Odenkirchen M., Brosche P., 1996, AG Abstr. Ser. 12, 191

Oja T., 1987, A\&AS 68, 211

Soma M., 1993, in: Mueller I.I., Kolaczek B. (eds.), IAU Symp. 156, Developments in Astrometry and their Impact on Astrophysics and Geodynamics. Kluwer, Dordrecht, p. 404

Tucholke H.-J., Brosche P., Odenkirchen M., 1996, A\&AS (accepted)

Turon C., et al., 1992, The Hipparcos Input Catalogue. ESA-SP 1136

Wielen R., 1995, A\&A 302, 613 